MATHEMATICS OF COMPUTATION

Volume 72, Number 243, Pages 1057-1066

S 0025-5718(02)01459-X

Article electronically published on November 18, 2002

\title{
A CONFORMING FINITE ELEMENT METHOD FOR OVERLAPPING AND NONMATCHING GRIDS
}

\author{
YUNQING HUANG AND JINCHAO XU
}

\begin{abstract}
In this paper we propose a finite element method for nonmatching overlapping grids based on the partition of unity. Both overlapping and nonoverlapping cases are considered. We prove that the new method admits an optimal convergence rate. The error bounds are in terms of local mesh sizes and they depend on neither the overlapping size of the subdomains nor the ratio of the mesh sizes from different subdomains. Our results are valid for multiple subdomains and any spatial dimensions.
\end{abstract}

\section{INTRODUCTION}

In recent years, there have been considerable interest in the use of overlapping grids. The main idea of overlapping grids is to divide a physical domain into a set of overlapping subregions which can accommodate smooth, simple, easily generated grids. In this approach, grid generation for complex geometries can be made simple, refinement grids can be added or removed without changing other grids, different equations/numerical methods may be used on different grids, and efficient structured grid solvers may be used. Furthermore, overlapping grids are well suited to parallelization and vectorization.

Overlapping grids have been much studied for finite difference as well as finite volume discretizations. For related works, we refer to [16, 17, 2, 20, 26, 14, 25, 26] and the references cited therein. As indicated in these references, the overlapping grids have been successfully applied in many engineering applications.

Recently, there have been some studies of applying the finite element method to overlapping grids. These studies are within the framework of mortar elements or Lagrange multipliers (refer to [13, 1, 23]). The mortar finite element method and more generally methods based on Lagrange multipliers have been mostly developed and extensively studied for the nonoverlapping nonmatching grids (cf. [10, 11, 27. $822,23,12,6,7,28$ ). In adaptive refinement of the finite element method, the nonmatching grids at the interfaces between the refined mesh and nonrefined mesh are treated by taking the values at the new grid points which are not previous grid

Received by the editor May 30, 2001 and, in revised form, November 7, 2001.

2000 Mathematics Subject Classification. Primary 65F10, 65N30.

Key words and phrases. Nonmatching grid, partition of unity, finite element, overlapping, domain decomposition, mortar.

The work was subsidized by the special funds for Major State Basic Research Projects through Xiangtan University, PRC, and partially supported by NSF DMS-0074299 through Pennsylvania State University and the Center for Computational Mathematics and Applications. 
points to be the interpolation of the values at "old" grid points (see [15]); this can be viewed as a special "mortar" in the nonoverlapping subdomain case.

In this paper, we present a new finite element discretization techniques that can be applied to general overlapping or nonmatching grids. Optimal error estimates are proved for the overlapping case with multiple subdomains. The nonoverlapping case can be carried out as a consequence of the overlapping case.

Our new finite element method admits an optimal error estimate for very minimal overlappings. In comparison, in the finite difference methods for overlapping grids and the convergence rate of the method, as observed and analyzed in [19, 14, depend crucially on the size of the overlapping regions. The optimal error estimates for our new method is also true for the multiple subdomain case. In comparison, the mortar finite element method does not always enjoy this optimal property (see 1]). In the mortar element approach, it is often a challenging task to impose matching conditions across different subdomains, and this kind of difficulty does not exist in our new approach.

Our new finite element discretization for overlapping grids is based on the partition of unity technique. The partition of unity method [4, 5] is frequently used implicitly or explicitly to develop so-called generalized finite element methods, including various "mesh-free" Galerkin methods [9, the $h p$ clouds [18], the reproducing kernel particle method [24], etc. For an overview see Babuška and Melenk [3] and Belytschko, Krongauz, Organ, Fleming and Krysl 9]. Huang and Xu [21] applied a partition of unity method to homogenization problems. It has been shown that the partition of unity method is a powerful tool for handling a large variation of problems efficiently. The main advantage is that it localized the approximation, which can be very flexible, and keeps the global continuity.

The study of the conforming finite element for overlapping and nonmatching grids is still in its infancy (although the main idea in this paper was already reported by the author at the Twelfth Domain Decomposition Meeting in Chiba, Japan in October 1999). The main pupose of this short paper is to set up a theoretical framework for the development of conforming finite element methods for overlapping and nonmatching grids. There are still many relevant practical issues that call for further studies on this method.

The rest of the paper is organized as follows. In Section 2, we shall give a brief description on overlapping grids and a simple naive finite element method associated with the overlapping grid. In Section 3, we present the main result of the paper: the construction of a new finite element space based on the partition of unity. In Section 4, we give a brief discussion of how our new method can be applied to nonoverlapping grids. Finally, in Section 5, we give a simple illustration of how our new method can be applied to elliptic boundary value problems.

\section{OVERLAPPING NONMATCHING GRIDS AND SUBDOMAIN SUBSPACES}

Let us first introduce some notation to be used in the paper. Given a bounded domain $\Omega \subset \mathbb{R}^{d}$, let $W^{k, p}(\Omega)$ be a $L^{p}$-based Sobolev space equipped with norms and seminorms

$$
\|u\|_{k, p, \Omega}=\left(\int_{\Omega} \sum_{|\alpha| \leq k}\left|D^{\alpha} u\right|^{p}\right)^{\frac{1}{p}}, \quad|u|_{k, p, \Omega}=\left(\int_{\Omega} \sum_{|\alpha|=k}\left|D^{\alpha} u\right|^{p}\right)^{\frac{1}{p}},
$$


where $\alpha=\left(\alpha_{1}, \alpha_{2}, \ldots, \alpha_{d}\right)$ is a multi-index. When $p=2$ we denote $W^{k, 2}(\Omega)$ by $H^{k}(\Omega)$ and omit the index $p$ in the norm notation. Given $\Gamma \subset \partial \Omega$, we denote by $H_{0}^{k}(\Omega ; \Gamma)$ the closure (in $H^{k}$ topology) of $C^{\infty}(\bar{\Omega})$ functions that vanish in a neighborhood of $\Gamma$. In particular, $H_{0}^{k}(\Omega)=H_{0}^{k}(\Omega ; \partial \Omega)$.

We shall use the notation $\lesssim, \gtrsim$, as in Xu [29, 30]; i.e., when we write $x_{1} \lesssim y_{1}$, $x_{2} \gtrsim y_{2}$, we mean there exist constants $c_{i}, i=1,2$ such that

$$
x_{1} \leq c_{1} y_{1}, \quad x_{2} \geq c_{2} y_{2},
$$

where $c_{i}$ 's are constants independent of mesh size $h$.

Let us now begin our discussion of overlapping grids. Given a bounded domain $\Omega \subset \mathbb{R}^{d}$, we consider an overlapping domain decomposition of $\Omega$; namely, we take $\Omega_{1}, \Omega_{2}, \ldots, \Omega_{p}$ to be overlapping subdomains satisfying

$$
\Omega=\bigcup_{i=1}^{p} \Omega_{i}
$$

Figure 1 is an illustration of overlapping grids in two dimensions.

We assume that each $\Omega_{i}$ is partitioned by a finite element triangulation $T^{h_{i}}$ of maximal mesh size $h_{i}$. We note that different $T^{h_{i}}$ on a different domain $\Omega_{i}$ may be completely independent of each other.

Associated with each triangulation $T^{h_{i}}$, we assume that we are given a finite element subspace $S^{h_{i}}\left(\Omega_{i}\right) \subset H^{r}(\Omega)$ (for some integer $r \geq 1$ ) having the following standard approximation properties: For any $u \in H^{m_{i}+r}\left(\Omega_{i}\right)$ (for some integers $\left.m_{i} \geq 1\right)$, there exists $v_{h} \in S^{h_{i}}\left(\Omega_{i}\right)$ such that

$$
\sum_{k=0}^{r} h_{i}^{k}\left|u-v_{h}\right|_{k, \Omega_{i}} \lesssim h_{i}^{m_{i}+r}\|u\|_{m_{i}+r, \Omega_{i}},
$$

and, for any $u \in H^{m_{i}+r}\left(\Omega_{i}\right) \cap H_{0}^{r}\left(\Omega_{i} ; \Gamma_{i}\right)$ (with $\Gamma_{i}$ being either $\partial \Omega_{i}$ or $\partial \Omega_{i} \backslash \partial \Omega$ ), there exists $v_{h} \in S^{h_{i}}\left(\Omega_{i}\right) \cap H_{0}^{r}\left(\Omega_{i} ; \Gamma_{i}\right)$ such that

$$
\sum_{k=0}^{r} h_{i}^{k}\left|u-v_{h}\right|_{k, \Omega_{i}} \lesssim h_{i}^{m_{i}+r}\|u\|_{m_{i}+r, \Omega_{i}} .
$$

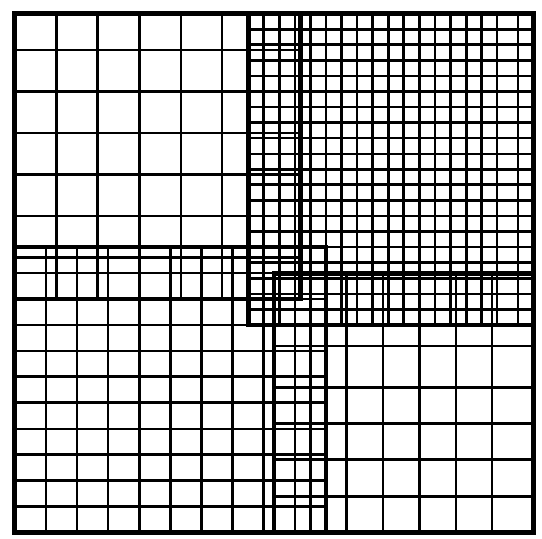

Figure 1. An illustrative picture of overlapping grids 
The main question we would like to ask is how to put together these local finite subspaces $S^{h_{i}}\left(\Omega_{i}\right)$ to construct a global finite element subspace of $H^{1}(\Omega)$. We would like to emphasize here that, unlike existing techniques such as Lagrange multiplier methods or mortar element methods, we are trying to construct a globally conforming finite element subspace in $H^{r}(\Omega)$.

The main ingredient in our analysis and construction below is a partition of unity $\left\{\vartheta_{i}\right\}$ associated with the overlapping subdomains $\left\{\Omega_{i}\right\}$ 's. It is easy to see that we can choose this partition of unity functions $\vartheta_{i}$ to satisfy the properties

$$
\left\{\begin{array}{l}
0 \leq \vartheta_{i} \leq 1, \\
\sum_{i} \vartheta_{i} \equiv 1, \\
\operatorname{supp}\left(\vartheta_{i}\right) \subset \bar{\Omega}_{i}, \vartheta_{i} \in W^{r, \infty}(\Omega), \\
\left|\nabla^{k} \vartheta_{i}\right| \lesssim d_{i}^{-k} \quad 1 \leq k \leq r,
\end{array}\right.
$$

where $d_{i}$ is the minimal overlapping size of $\Omega_{i}$ with its neighboring subdomains.

To gain some insight, let us first describe a naive approach to construct a globally conforming finite element space. The idea is simply to enforce a function in $S^{h_{i}}\left(\Omega_{i}\right)$ to be zero on $\partial \Omega_{i} \backslash \partial \Omega$ and to use the zero extension of such a function to the rest of the domain. Namely we define

$$
\hat{S}^{h_{i}}\left(\Omega_{i}\right)=\left\{v \in H^{r}(\Omega),\left.v\right|_{\Omega_{i}} \in S^{h_{i}}\left(\Omega_{i}\right) \text { and } v=0 \text { on } \Omega \backslash \Omega_{i}\right\} .
$$

Because each such space is globally defined as a subspace of $H^{r}(\Omega)$, we can then naturally put them together by summing them up:

$$
S^{h}(\Omega)=\sum_{i=1}^{p} \hat{S}^{h_{i}}\left(\Omega_{i}\right)
$$

For the space defined above, we have the following simple result.

Theorem 1. For any given $u \in H^{r}(\Omega) \bigcap_{i=1}^{p} H^{m_{i}+r}\left(\Omega_{i}\right)$,

$$
\inf _{v_{h} \in \hat{S}^{h}(\Omega)}\left\|u-v_{h}\right\|_{r, \Omega} \lesssim \sum_{i=1}^{p} d_{i}^{-\left(m_{i}+r\right)} h_{i}^{m_{i}}\|u\|_{m_{i}+r, \Omega_{i}} .
$$

Proof. Given $u \in H^{r}(\Omega) \bigcap_{i=1}^{p} H^{m_{i}+r}\left(\Omega_{i}\right)$, let $u_{i}=\vartheta_{i} u \in H_{0}^{r}\left(\Omega_{i} ; \partial \Omega \backslash \partial \Omega_{i}\right)$, where $\left\{\vartheta_{i}\right\}$ is a partition of unity satisfying (3). By (2), there exists $v_{i} \in S^{h_{i}}\left(\Omega_{i}\right) \cap$ $H_{0}^{r}\left(\Omega_{i} ; \partial \Omega \backslash \partial \Omega_{i}\right)$ such that

$$
\left\|u_{i}-v_{i}\right\|_{r, \Omega_{i}} \lesssim h_{i}^{m_{i}}\left|u_{i}\right|_{m_{i}+r, \Omega_{i}}
$$

Let $\hat{v}_{i} \in \hat{S}^{h_{i}}\left(\Omega_{i}\right)$ be the zero extension of $v_{i}$ and set $v_{h}=\sum_{i=1}^{p} \hat{v}_{i}$. We have

$$
\begin{aligned}
\|u-v\|_{r, \Omega} & =\left\|\sum_{i=1}^{p}\left(u_{i}-\hat{v}_{i}\right)\right\|_{r, \Omega} \\
& \leq \sum_{i=1}^{p}\left\|u_{i}-v_{i}\right\|_{r, \Omega_{i}} \lesssim \sum_{i=1}^{p} h_{i}^{m_{i}}\left|u_{i}\right|_{m_{i}+r, \Omega_{i}} \\
& \lesssim \sum_{i=1}^{p} d_{i}^{-(m+r)} h_{i}^{m_{i}}\|u\|_{m_{i}+r, \Omega_{i}} .
\end{aligned}
$$

This completes the proof. 
More refined analysis in some special cases. In some special situations, the error estimates obtained above can be slightly improved. For example, if the size of overlapped region, denoted, say, by $G_{i}$, in each $\Omega_{i}$ is of order $O\left(d_{i}\right)$, then we can estimate $\left|u_{i}\right|_{m_{i}+r, \Omega_{i}}$ as

$$
\begin{aligned}
\left|u_{i}\right|_{m_{i}+r, \Omega_{i}} & \lesssim \sum_{k=1}^{m_{i}+r}\left\|\nabla^{k} \theta_{i}\right\|_{0, \infty, G_{i}}\|u\|_{m_{i}+r-k, G_{i}}+|u|_{m_{i}+r, \Omega_{i}} \\
& \lesssim \sum_{k=1}^{m_{i}+r} d_{i}^{-k}\|u\|_{m_{i}+r-k, \Omega_{i}}+|u|_{m_{i}+r, \Omega_{i}} \\
& \lesssim d_{i}^{-\left(m_{i}+r\right)+1}\left(\|u\|_{0, \infty, \Omega_{i}}+\|u\|_{m_{i}+r, \Omega_{i}}\right) .
\end{aligned}
$$

In this case, we have a slightly improved error estimate (comparing Theorem 11):

$$
\inf _{v_{h} \in \hat{S}^{h}(\Omega)}\left\|u-v_{h}\right\|_{r, \Omega} \lesssim \sum_{i=1}^{p} d_{i}^{-\left(m_{i}+r\right)+\frac{1}{2}} h_{i}^{m_{i}}\left(\|u\|_{0, \infty, \Omega_{i}}+\|u\|_{m_{i}+r, \Omega_{i}}\right) .
$$

This appears to be the best estimate we can obtain for this situation without making any further assumption about how the grids from different subdomains are overlapped. In the most trivial situation when we have a global conforming grid, we can also view this global grid as an overlapping grid. In this case, we can of course get the usual optimal error estimate that is certainly better than the above one. But this trivial case is not interesting.

Then one interesting question is whether it is possible to obtain an optimal error estimate for nontrivial overlapping grids. The answer to this question is, in general, negative. Let us now give a counterexample to illustrate this.

Let us consider a one dimensional problem with $\Omega=(-1,1)$ and the two subdomains

$$
\Omega_{1}=(-1, h) \quad \text { and } \quad \Omega_{2}=(0,1) .
$$

We further assume that the two right-most grid points in $\bar{\Omega}_{1}$ are $x=-h$ and $x=h$, and the two left-most grid points in $\bar{\Omega}_{2}$ are $x=0$ and $x=h$. We note that $x=0$ is not a grid from the local finite element space in $\Omega_{1}$.

In this case, we claim the resulting (say, linear finite element) space as defined in (4) cannot lead to an optimal error estimate. This is because the constant function in $(-h, h)$ cannot be recovered from the resulting space. We can draw similar conclusions for the cases with more elements overlapped. For example, suppose that the left boundary point of the right subdomain is located in the inside of an element of the left subdomain, and make the same assumptions on the left subdomain. Then the constant function on the region of overlapping parts and the two elements containing the boundary points of the subdomains cannot be recovered. But we can find that a proper approximation can be derived, which is more accurate as the overlapping part gets bigger.

\section{Construction of a global conforming subspace USING THE PARTITION OF UNITY}

Not surprisingly, as shown in Theorem 1, the approximation properties from the space obtained by the naive approach deteriorates as the overlapping size decreases. In this section, we shall employ a new technique based on the partition of unity to 
construct a globally conforming finite element space that has optimal approximation properties.

The idea is actually quite simple, namely to use the partition of unity as described in (3) to glue all the local subspaces $S^{h_{i}}\left(\Omega_{i}\right)$ together. More specifically, we define

$$
V^{h}(\Omega)=\sum_{i=1}^{p} \vartheta_{i} S^{h_{i}}\left(\Omega_{i}\right)=\left\{v=\sum_{i=1}^{p} \vartheta_{i} v_{i}, \quad v_{i} \in S^{h_{i}}\left(\Omega_{i}\right)\right\} .
$$

Then we have the following optimal error estimates.

Theorem 2. Assume the overlapping size $d_{i} \geq c h_{i}$. Then for any $u \in$ $H^{r}(\Omega) \bigcap_{i=1}^{p} H^{m_{i}+r}\left(\Omega_{i}\right)$,

$$
\inf _{v_{h} \in V^{h}(\Omega)}\left\|u-v_{h}\right\|_{k, \Omega} \lesssim \sum_{i=1}^{p} h_{i}^{m_{i}+r-k}\|u\|_{m_{i}+r-k, \Omega_{i}}, \quad 0 \leq k \leq r .
$$

Proof. Thanks to (1), we can choose $v_{i} \in S^{h_{i}}\left(\Omega_{i}\right)$ satisfying

$$
\sum_{k=j}^{r} h_{i}^{k}\left|u-v_{i}\right|_{j, \Omega_{i}} \lesssim h_{i}^{m_{i}+r}|u|_{m_{i}+r, \Omega_{i}} .
$$

Setting $v=\sum_{i=1}^{p} \vartheta_{i} v_{i}$, we have

$$
\begin{aligned}
\left\|u-v_{h}\right\|_{r, \Omega} & =\left\|\sum_{i=1}^{p} \vartheta_{i} u-\sum_{i=1}^{p} \vartheta_{i} v_{i}\right\|_{r, \Omega} \\
& \leq \sum_{i=1}^{p}\left\|\vartheta_{i}\left(u-v_{i}\right)\right\|_{r, \Omega_{i}} \\
& \lesssim \sum_{i=1}^{p} \sum_{j=0}^{r} d_{i}^{j-r}\left\|u-v_{i}\right\|_{j, \Omega_{i}} \\
& \lesssim \sum_{i=1}^{p} \sum_{j=0}^{r} h_{i}^{j-r}\left\|u-v_{i}\right\|_{j, \Omega_{i}} \\
& \lesssim \sum_{i=1}^{p} h_{i}^{m_{i}}|u|_{m_{i}+r, \Omega_{i}} .
\end{aligned}
$$

This completes the proof for $j=r$. The proof for other cases is obviously similar.

Remark 1 . The condition $d_{i} \gtrsim h_{i}$ means that the overlapping size in $\Omega_{i}$ should at least have the width $h_{i}$, which is the grid size. This is a very weak assumption for overlapping grids.

Remark 2. In the above construction and analysis, we may also allow $r$ to vary on different subdomains. Such a more general choice of $r$ may be useful when different types of partial differential equations are used in different subdomains.

\section{NONOVERLAPPING NONMATCHING GRIDS}

In the previous section we proposed a generalized finite element method for overlapping nonmatching grids. The error estimates show that the error bound is optimal if the overlap is not less than the grid size in each subdomain. As a 


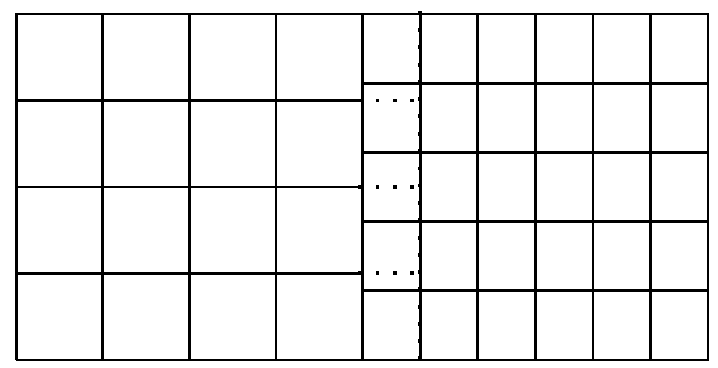

Figure 2. Creating overlapping grids by simple extension

result we cannot take the limit to obtain the nonoverlapping nonmatching grid finite element method by letting the overlapping size tend to zero. In this section, we shall discuss a simple technique to deal with this situation.

Without loss of generality, let us consider two subdomain cases. The analysis for many subdomain cases is similar.

Let $\Gamma$ be the interface of $\Omega_{1}$ and $\Omega_{2}$, and $T^{h_{1}}$ and $T^{h_{2}}$ are triangulations on $\Omega_{1}$ and $\Omega_{2}$ independently. The grid on the interface $\Gamma$ generally does not match each other. Let us suppose $h_{1} \geq h_{2}$ and extend the mesh in $\Omega_{1}$ to $\Omega_{2}$ for one more element. So we get overlapping subdomains and nonmatching grids $\widetilde{T}^{h_{1}}$ and $T^{h_{2}}$ (see a typical example in Figure 2 for two dimensional case). Let $\Gamma^{\prime} \subset \Omega_{2}$ be the boundary of $\tilde{\Omega}_{1}$, then

$$
\operatorname{dist}\left(\Gamma, \Gamma^{\prime}\right) \gtrsim h_{1} \geq h_{2} .
$$

Denote the nodes of $\widetilde{T}^{h_{1}}\left(\tilde{\Omega}_{1}\right)$ on $\Gamma^{\prime}$ by $\widetilde{x}_{j}$, the unknowns by $\widetilde{u}_{j}$.

Let $\theta_{1}$ and $\theta_{2}$ be partition of unity such that

$$
\theta_{1}(\Gamma)=1, \quad \theta_{1}\left(\Gamma^{\prime}\right)=0, \quad \theta_{2}(\Gamma)=0, \quad \theta_{2}\left(\Gamma^{\prime}\right)=1,
$$

and $\theta_{1}=1$ on the left of $\Gamma$ and $\theta_{2} \theta=1$ on the right of $\Gamma^{\prime}$.

Eliminating the unknowns $\widetilde{u}_{j}$, we derive a system which is equivalent to the nonoverlapping grid finite element system plus a constraint to the unknowns near the interface. This constraint can be regarded as a special kind of "mortar". The error estimates can be obtained by the overlapping case.

\section{Applications to elliptic boundary Value problems}

With the optimal approximation property (6), the globally conforming finite element space defined in (5) can naturally be applied for discretization of (both steady and nonsteady state) partial differential equations of various kinds. In this section, let us give an illustration of its application to elliptic boundary value problems.

We shall consider general elliptic boundary value problems (including systems) in $d$-dimension. Let $\Omega \subset \mathbb{R}^{d}$ be a bounded domain and $V(\Omega)=\left[H_{0}^{r}(\Omega)\right]^{n}$, where $n \geq 1$ (depending on whether $u$ is scalar or vector).

Let $a(\cdot, \cdot)$ be a bilinear form defined on $V(\Omega) \times V(\Omega)$ as

$$
a(u, v)=\sum_{|\alpha|=r,|\beta|=r}\left(a_{\alpha, \beta} D^{\alpha} u, D^{\beta} v\right)_{0, \Omega}+\left(L_{0} u, v\right)_{0, \Omega},
$$

where $L_{0}$ is a linear partial differential operator of order less than $r$. 
Obviously $a(\cdot, \cdot)$ is a continuous bilinear form on $V(\Omega) \times V(\Omega)$. We further assume that the following inf-sup conditions are satisfied:

$$
\inf _{u \in V(\Omega)} \sup _{v \in V(\Omega)} \frac{|a(u, v)|}{\|u\|_{r, \Omega}\|v\|_{r, \Omega}}>0, \quad \inf _{v \in V(\Omega)} \sup _{u \in V(\Omega)} \frac{|a(u, v)|}{\|u\|_{r, \Omega}\|v\|_{r, \Omega}}>0 .
$$

We then have the following well-posed variational problem: Find $u \in V(\Omega)$ such that

$$
a(u, v)=\langle f, v\rangle \quad \forall v \in V(\Omega),
$$

for $f \in\left[H^{-r}(\Omega)\right]^{n}$.

With the generalized finite element space given by (5), we have the finite element discretization of (8) as follows: Find $u_{h} \in V_{h}(\Omega)$ such that

$$
a\left(u_{h}, v_{h}\right)=\left\langle f, v_{h}\right\rangle \quad \forall v_{h} \in V_{h}(\Omega) .
$$

By the approximation estimate ([6), we can use standard techniques to obtain the following result.

Theorem 3. If $\max _{1 \leq i \leq p} h_{i}$ is sufficiently small, the discrete problem (91) is well posed and its solution $u_{h}$ admits the error estimate

$$
\left\|u-u_{h}\right\|_{r, \Omega} \lesssim \inf _{v_{h} \in V_{h}(\Omega)}\left\|u-v_{h}\right\|_{r, \Omega} \lesssim \sum_{i=1}^{p} h_{i}^{m_{i}}\|u\|_{m_{i}+r, \Omega_{i}} .
$$

The above examples are stated only for pure Dirichlet problems. If we use $H^{r}(\Omega)$ in replace of $H_{0}^{r}(\Omega)$, we would get pure Neumann problems. We can of course also consider mixed boundary value problems.

Let us take a special look at the case $r=1$. In this case, we have second order elliptic boundary value problems with the error estimate

$$
\left\|u-u_{h}\right\|_{1, \Omega} \lesssim \sum_{i=1}^{p} h_{i}^{m_{i}}\|u\|_{m_{i}+1, \Omega_{i}} .
$$

As in the standard finite element method, we can also derive the error estimate in the $L^{2}$ norm. For example, if the coefficients in the underlying partial differential equations are adequately smooth and if either $\partial \Omega$ is smooth or $\Omega$ is convex, we can use a standard duality argument to obtain the error estimate

$$
\left\|u-u_{h}\right\|_{0, \Omega} \lesssim\left(\max _{1 \leq j \leq p} h_{j}\right) \sum_{i=1}^{p} h_{i}^{m_{i}}\|u\|_{m_{i}+1, \Omega_{i}} .
$$

\section{Some COMments on the IMPLEMENTATiON}

In this section, we give brief a discussion on implementation issues of the proposed method.

Overlapping case. The implementation issue has been much studied for the finite difference or finite volume methods for overlapping grids (e.g., [16, 17]) and the techniques developed before can also be applied to our new proposed finite element methods. The key point is that, in the framework of overlapping grids, the local grids are assumed to be structured (mostly topologically Cartesian grids). In fact one main reason that we use overlapping grids is to be able to use local structured grids. 
Without loss of generality, we can get the main idea by studying the implementation issue on overlapped Cartesian grids. The extension to more general structured grids can be realized with some proper isomorphic mappings with the Cartesian grids.

One main issue in the implementation of our proposed method is to decide the relevant location of each grid point or each element from one grid to another grid. But this is quite straightforward for Cartesian grids by simply checking the coordinates of the given grid point (or vertices of the given element) and the relevant locations of each (topologically structured) subdomain that is involved. For the purpose of numerical integration, we also need to know how elements from different subdomains intersect each other. This can be rather messy, but this is a price that we need to pay for this type of method. In practice, the overlapped region has a very small measure and these types of elements are not that many (typically the number of such elements should be of order $O(n)$ if the total number of elements is of order $O\left(n^{d}\right)(d=2,3)$ in $d$-dimensional space). The key point again is that we are working with piece-wise Cartesian grids, so the computational cost is quite moderate.

Nonoverlapping case. We feel that our proposed algorithm is particularly interesting for the nonoverlapping nonmatching grids (see Section 4 ). Here, let us discuss the specific issue of implementation; namely the construction of the partition of unity. We observe that one natural construction where $\theta_{1}$ and $\theta_{2}$ satisfy (77) is to use the element shape function with the grid on the extended overlapping region. Namely, we take $\theta_{1}$ to be the summation of all nodal basis functions (linear or bilinear) associated with all the grid points on $\Gamma$ and, similarly, we take $\theta_{2}$ to be the summation of all nodal basis functions associated with all the grid points (from the extended grid) on $\Gamma^{\prime}$.

\section{REFERENCES}

1. Y. Achdou and Y. Maday. The mortar element method with overlapping subdomains. In Proceedings of the 12th International Conference on Domain Decomposition Methods. www.ddm.org. CMP 2001:12

2. M. Aftosmis, J. Melton, and M. Berger. Adaptation and surface modeling for cartesian mesh methods. In 12th AIAA CFD. Conf, volume AIAA Paper 95-1725, San Diego, CA, June 1995.

3. I. Babuška and J. M. Melenk. The partition of unity finite element method: Basic theory and applications. Comp. Meth. Appl. Mech. Engrg., 139:289-314, 1996. MR 97k:65258

4. I. Babuška and J. M. Melenk. The partition of unity method. Internat. J. Numer. Methods Engrg., 40:727-758, 1997. MR 97j:73071

5. I. Babuška and Z. Zhang. The partition of unity method for the elastically supported beam. Comp. Meth. Appl. Mech. Engrg., 152:1-18, 1998. MR 99b:73039

6. F. B. Belgacem. The mortar finite element method with lagrange multipliers. Numer. Math., 1998.

7. F. B. Belgacem. The mortar finite element method with lagrange multiplierss. Numer. Math., pages 173-197, 1999.

8. F. B. Belgacem and Y. Maday. The mortar element method for three dimensional finite elements. $M^{2} A N, 31: 289-302,1997$.

9. T. Belytschko, Y. Krongauz, D. Organ, M. Fleming, and P. Krysl. Meshless methods: An overview and recent developments. Comp. Meth. Appl. Mech. Engrg., 139:3-48, 1996.

10. C. Bernardi, Y. Maday, and A. T. Patera. Domain decomposition by the mortar element method. In H. Kaper et al., editor, Asymptotic and numerical methods for partial differential equations and their applications, pages 269-286, Dordrecht, 1993. Reidel. MR 94c:65151 
11. C. Bernardi, Y. Maday, and A. T. Patera. A new nonconforming approach to domain decomposition: the mortar element method. In Nonlinear partial differential equations and their applications, pages 13-51, Paris, 1994. MR 95a:65201

12. D. Braess and W. Dahmen. Stability estimates of the mortar finite element method for 3dimensional problems. East-West J. Numer. Math., pages 249-263, 1998. MR 2000e:65115

13. X.-C. Cai, M. Dryja, and M. Sarkis. Overlapping non-matching grids mortar element methods for elliptic problems. SIAM J. Numer. Anal., 36(2):581-606, 1999. MR 2000a:65142

14. X.-C. Cai, T. Mathew, and M. Sarkis. Maximun norm analysis of overlapping non-matching grids discretization of elliptic problems. SIAM J. Numer. Anal., 37(5):1709-1728. MR 2001e:65130

15. Y. Chen and Y. Huang. The superconvergence and asymptotic exact a posteriori error estimates of finite element on k-mesh. Numer. Math. Sinica, 16:278-285, 1994. MR 97c:65184

16. G. Chesshire and W. Henshaw. Composite overlapping meshes for the solution of partial differential equations. J. Comp. Phys., 90:1-64, 1990.

17. W. D. Henshaw D. L. Brown and D. J. Quinlan. Overture: An object-oriented framework for solving partial differential equations on overlapping grids. Technical report, UCRL-JC-132017, 1999.

18. C. A. M. Duarte and J. T. Oden. $h p$ clouds-an $h p$ meshless method. Numer. Meth. Partial Diff. Eqns., 12:673-705, 1996. MR 98a:65163

19. W. Henshaw. Part I: The numerical solution of hyperbolic systems of conservation laws; Part II: Composite overlapping grid techniques. PhD thesis, Dept. Appl. Math., California Institute of Technology, Pasadena, CA, 1985.

20. W. Henshaw. A fourth-order accurate method for the incompressible Navier-Stokes equations on overlapping grids. J. Comp. Phys., 113:13-25, 1994. MR 95b:76066

21. Y. Huang and J. Xu. Convergence of a generalized finite element method for elliptic problems with highly oscillating coefficients. Manuscript.

22. Y. Kuznetsov. Efficient iterative solvers for elliptic finite element problems on non-matching grids. Russian J. Numer. Anal. Math. Modelling, 10:187-211, 1995. MR 96j:65125

23. Y. Kuznetsov. Overlapping domain decomposition with non-matching grids. In P. Bjostad, M. Espedal, and D. Keyes, editors, Proceedings of the 9th International Conference on Domain Decomposition, pages 64-76. Domain Decomposition Press, 1998.

24. W. K. Liu, S. Jun, J. Adee, and T. Belytschko. Reproducing kernel particle methods for structural dynamics. Internat. J. Numer. Methods Engrg., 38:1655-1679, 1995. MR 96b:73112

25. G. Starius. Composite mesh difference methods for elliptic problems. Numer. Math., 28:243258, 1977. MR 57:1923

26. J. Steger and J. Benek. On the use of composite grid schemes in computational aerodynamics. Comp. Meth. Appl. Mech. Engrg., (64):301-320, 1987. MR 88i:65146

27. P. Le Tallec, T. Sassi and M. Vidrascu. Three-dimensional domain decomposition methods with nonmatching grids and unstructured coarse solvers. In D. Keyes and J. Xu, editors, Prceedings of the 7th international conference on domain decomposition, Comtemp. Math., pages 61-64. AMS, 1994. MR 95j:65167

28. B. I. Wohlmuth. Discretization methods and iterative solvers based on domain decomposition. Springer, 2001. MR 2002c:65231

29. J. Xu. Theory of Multilevel Methods. PhD thesis, Cornell University, 1989.

30. J. Xu. Iterative methods by space decomposition and subspace correction. SIAM Review, 34:581-613, 1992. MR 93k:65029

Institute for Computational and Applied Mathematics and Department of Mathematics, Xiangtan University, Peoples Republic of China, 411105

E-mail address: huangyq@mail.xtu.edu.cn

Center for Computational Mathematics and Applications Pennsylvania State UniVersity, University Park, Pennsylvania 16803

E-mail address: xu@math.psu.edu, http://www.math.psu.edu/xu 